\title{
The emergence of superconducting systems in Anti-de Sitter space
}

\author{
W.M. Wu, ${ }^{a}$ M.P. Pierpoint, ${ }^{b}$ D.M. Forrester ${ }^{c, 1}$ and F.V. Kusmartsev ${ }^{a}$ \\ ${ }^{a}$ Department of Physics, Loughborough University, \\ Loughborough LE11 3TU, U.K. \\ ${ }^{b}$ Institute of Physics and Chemistry, Ogarev Mordovia State University, \\ Saransk, 430005, Russia \\ ${ }^{c}$ Department of Chemical Engineering, Loughborough University, \\ Loughborough LE11 3TU, U.K. \\ E-mail: W.M.Wu2@lboro.ac.uk, M.Pierpoint@lboro.ac.uk, \\ D.M.Forrester@lboro.ac.uk, F.Kusmartsev@lboro.ac.uk
}

ABSTRACT: In this article, we investigate the mathematical relationship between a $(3+1)$ dimensional gravity model inside Anti-de Sitter space $\mathrm{AdS}_{4}$, and a $(2+1)$ dimensional superconducting system on the asymptotically flat boundary of $\mathrm{AdS}_{4}$ (in the absence of gravity). We consider a simple case of the Type II superconducting model (in terms of Ginzburg-Landau theory) with an external perpendicular magnetic field $\mathbf{H}$. An interaction potential $V(r, \psi)=\alpha(T)|\psi|^{2} / r^{2}+\chi|\psi|^{2} / L^{2}+\beta|\psi|^{4} /\left(2 r^{k}\right)$ is introduced within the Lagrangian system. This provides more flexibility within the model, when the superconducting system is close to the transition temperature $T_{c}$. Overall, our result demonstrates that the Ginzburg-Landau differential equations can be directly deduced from Einstein's theory of general relativity.

KeYwords: Holography and condensed matter physics (AdS/CMT), Classical Theories of Gravity, Duality in Gauge Field Theories

ARXIV EPRINT: 1605.04092

\footnotetext{
${ }^{1}$ Corresponding author.
} 


\section{Contents}

1 Introduction 1

2 Gravity model 3

3 Superconducting system 5

4 Methodology and analysis $\quad 6$

$\begin{array}{ll}4.1 \text { Coupling differential equations } & 7\end{array}$

$\begin{array}{lll}4.2 & \text { Approximated solutions } & 7\end{array}$

4.3 At the infinite boundary of AdS space: $r \rightarrow \infty \quad 7$

4.3.1 First scalar solution $\psi_{1} / r\left(\right.$ set $\left.\psi_{2}=0\right) \quad 7$

4.3.2 Second scalar solution $\psi_{2} / r^{2}\left(\right.$ set $\left.\psi_{1}=0\right) \quad 8$

4.4 The physical meaning of $\psi \quad 9$

5 Conclusion and remarks $\quad 9$

A Ginzburg-Landau equations from thermodynamic approach $\quad 10$

\section{Introduction}

Since the discovery of superconductivity in 1911 by Kamerlingh Onnes, theories describing the associated phenomena have been under constant modification and evolution. The explanation of "elemental" superconducting behaviour (e.g. in metals such as lead and aluminium), with zero resistivity at temperatures close to zero kelvin in low magnetic fields, is well established. However, a different subset of superconductors (e.g. ceramics such as yttrium barium copper oxide and many other cuprates) exists that has much higher transition temperatures ( $>30 \mathrm{~K}$, at which point superconductivity breaks down) for which many physical mechanisms are not yet understood.

The first high temperature superconductor was discovered in 1986 by Bednorz and Müller [1]. The thermodynamic Ginzburg-Landau theory that is reasonably successful at describing high temperature superconductivity near the critical transition temperature $T_{c}$ was originally a phenomenological theory, but was later derived microscopically by Gor'kov [2] in 1959. Ginzburg and Landau defined superconductivity mathematically through the introduction of a complex order parameter field - a macroscopic coherent wave function $\psi$ - that appears below $T_{c}$. The free energy of a superconductor was expressed in its terms. The onset of superconductivity is a second order transition, i.e. there is no latent heat; the order parameter increases continuously from zero. Ginzburg-Landau theory follows from the general theory of Landau for second order phase transitions. It 
remarkably predicted the existence of high-temperature superconductors, whose properties include the penetration of magnetic flux into the structure in cylindrical tubes, called vortices [3] (this does not happen in elemental superconductors, where a magnetic field is expelled from the bulk - the Meissner effect).

The lattice structures of superconducting cuprates or pnicides are characterised by complex competing electronic and magnetic phases that emerge in association with fractal structures that develop from the nano level, and propagate up to many micrometres in size [4]. To create a high temperature superconductor one needs to dope a parent compound such as $\mathrm{La}_{2} \mathrm{CuO}_{4}$ with, for example, oxygen interstitials or strontium. The doping that creates the highest critical temperature is called optimal doping. At this optimal level a single $T_{c}$ value marks the transition to a superconducting phase. However, careful annealing to avoid the escape of interstitial oxygen produces a mixed state that can even have two critical temperatures [4]. This is caused by the self-organisation of the oxygen into different patterns, such as stripes [5], or the formation of dipolar resonance plaquettes [6]. Thus, a 1 or $2 D$ ordering of electronic density in high temperature superconductors may dictate the properties of the phase diagram and in particular the superconducting phase. At the optimal doping a continuous phase change - with a quantum critical state - may be realised at the transition point [7]. In these high temperature superconductors electron pairs form, but the mechanisms are quite different from conventional superconductors (that generally have much lower critical temperatures). There is a strong coupling mechanism (that is not due to phonons) involved that renders well-known theories that describe the conventional electron-phonon mechanism via the BCS model (after Bardeen Cooper and Schrieffer) unable to describe the physical properties. Conventional superconductivity typically involves pairs of electrons that are separated over distances larger than the lattice spacing, leading to a relatively weak binding. Thus, new methods of analysis are required in condensed matter physics that can lead to greater understanding of the complex issue of strongly correlated systems.

Here, we make use of techniques borrowed from cosmology that are valid for describing a superconductor when its temperature is equivalent to that of a corresponding black hole [8]. The fractal structures found in the high $T_{c}$ superconductors contain information about the origin and history of the sample. It was found in the experiments by Bianconi et al., that the development of granular fractal structures stimulated the onset of high temperature superconductivity (see, [9] and references therein). There are also long term discussions about the formation of the one-dimensional conducting channels, which may be associated with oxygen defects that lead in turn to the formation of Luttinger liquid inside these channels $[10,11]$. There the electron and spin degrees of freedom are decoupled and when channels are ordered, due to the Coulomb interaction, the charge-density wave (CDW) state develops [12]. However, the transverse stripes fluctuations suppress the CDW, increase the tunnelling between stripes and may create electron liquid crystal nematics which may contain a novel state of $2 \mathrm{D}$ Luttinger Liquid [12-14]. The conducting filaments of the electron nematic, in general, may form a critical state of some sort of fractal $[4,9]$ or the the electron spider web, where there is $2 \mathrm{D}$ conformal invariance and therefore the methods such as AdS/CFT correspondence may be applied. 
The fractal electron spider web or electron nematic might play a key role in the mechanism of the superconductivity in cuprates. It was also recently noticed that the superconductivity is enhanced near nematic quantum critical point $(Q C P)$ (see, the recent discussion in the ref. [15]) that may be associated with optimal doping. Likewise, a black hole's information is contained in threads or "hair" at its event horizon that grow from its time of formation, and its later development.

To use the cosmological models we need to mathematically create a black hole that has hair below $T_{c}$. The emergence of the superconducting phase corresponds to a black hole formed in Anti de Sitter (AdS) space [16-19] with hair [20]. A QCP is suspected to lie within the superconducting phase and quantum fluctuations are thought to extend its presence to temperatures well above absolute zero. Near $T_{c}$ the quantum fluctuations should be detectable throughout the superconducting condensate, with analogy to a black hole with the same quantum hair (i.e. entropy, information, temperature) [21]. Recent work in the iron pnictide superconductors [22] has found a $Q C P$ where the London penetration depth increases as a consequence of quantum fluctuations. A further signature of a $Q C P$ is superconductivity and magnetism coexisting as a consequence of doping. A possible material for demonstration of this phenomena is the new material with anomalous magnetoresistance, $\mathrm{LiTi}_{2} \mathrm{O}_{4}$ [23]. The work we develop herein may be useful in further developing the understanding of the physics of these novel materials. Theories derived from cosmology, e.g. AdS/conformal field theory, have previously been used in the description of cuprate superconductors in analogy to special black holes [9, 24]. Indeed the connection of superconducting fluids and superconductors with experiments to deduce cosmological mysteries is not new: for example, in 1985 Zurek proposed superconducting liquid helium as a possible laboratory test for the Kibble mechanism [25], with the theoretical description coming through Ginzburg-Landau theory.

The high $T_{c}$ superconductors are layered and can be described by $(2+1)$ dimensional models. Using the AdS gravitational model, the properties in the vicinity of $(2+1)$ quantum critical points may be investigated by finding a $(3+1)$-dimensional gravitational dual of the $(2+1)$ dimensional system below $T_{c}$. The AdS space is becoming an increasingly valuable tool in different branches of physics - including cosmology, string theories [26-32], condensed matter physics [33-36], and more recently, within the holographic principle $[26,37-40]$. AdS space has a negative curvature, conveniently offering resolution to the problem of the thermodynamically unstable Schwarzschild black-hole, as it possesses negative heat capacity [39, 40]. Thus, we provide a new methodology that demonstrates that the application of an AdS space within Einstein's theory of relativity can lead to the emergence of a superconducting system (i.e., Ginzburg-Landau theory [3, 41]), which is located on the AdS infinite boundary [33-36].

\section{Gravity model}

The line element of $\mathrm{AdS}_{4}$ is given by [36]

$$
d s^{2}=g_{\mu \nu} d x^{\mu} d x^{\nu},
$$




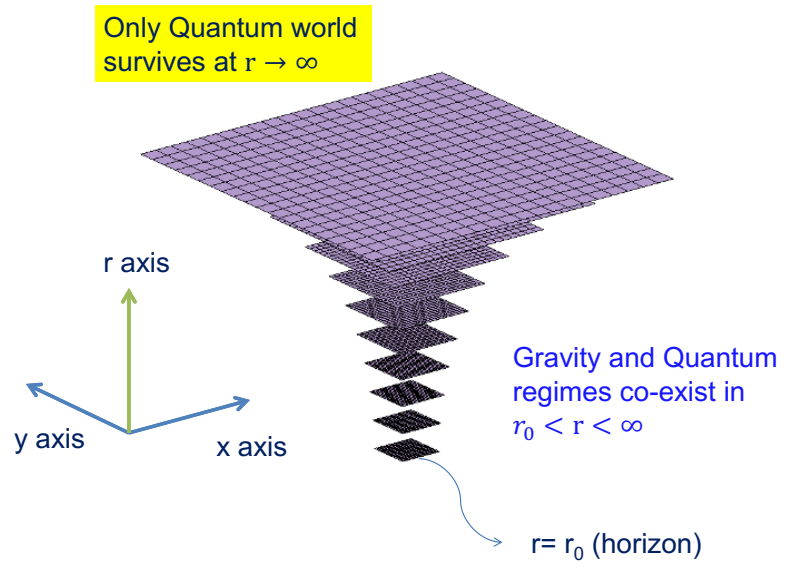

Figure 1. The spatial geometry in Anti-de Sitter (AdS) space with coordinates $(r, x, y)$ is shown. Here, $r$ represents the holographic axis, with only quantum phenomena surviving on the asymptotically flat ( $\mathrm{x}-\mathrm{y}$ plane) at $r \rightarrow \infty$.

with the system defined by Poincare coordinates $x^{\mu}=\{t, r, x, y\}$ [42]. The metric $g_{\mu \nu}$ is chosen as

$$
g_{\mu \nu}=\left(\begin{array}{cccc}
-s(r) & 0 & 0 & 0 \\
0 & 1 / s(r) & 0 & 0 \\
0 & 0 & r^{2} & 0 \\
0 & 0 & 0 & r^{2}
\end{array}\right)
$$

with the function $[34-36]$

$$
s(r)=\frac{r^{2}}{L^{2}}-\frac{r_{0}^{3}}{L^{2} r} .
$$

Here $r_{0}$ represents the horizon radius of the AdS black-hole, and is directly related to its mass. To be consistent with dimensionality, the characteristic AdS length scale $L=$ $\sqrt{-3 / \Lambda}$ has been re-parametrized in terms of the cosmological constant $\Lambda[34,36]$. We also note that the function $s(r)$ is not chosen arbitrarily, but rather as an explicit solution to the Einstein equations [34-36]. Both the AdS length scale $L$ and horizon radius $r_{0}$ determine the black-hole temperature $T=\frac{3 r_{0}}{4 \pi L^{2}}$, as mentioned by Hawking $[17,36]$.

The scale of the $\mathrm{x}-\mathrm{y}$ coordinate plane increases with the square of the holographic dimension $r$, namely $r^{2}\left(d x^{2}+d y^{2}\right)$. Assuming a stationary system with negligible backreaction, the spatial geometry of AdS can be realized as in figure 1. Back-reaction here refers to the curvature of space-time induced by small particles.

The required action for the gravity model is given by $S=\int \mathcal{L} \sqrt{|g|} \mathrm{d}^{4} x$, where the Lagrangian density $\mathcal{L}[35,43-45]$ is given by

$$
\mathcal{L}=\frac{1}{2 \kappa}\left(\mathcal{R}+\frac{6}{L^{2}}\right)+\mathcal{L}_{m}
$$

and

$$
\mathcal{L}_{m}=-\frac{1}{4 \mu_{0}} F_{\mu \nu} F^{\mu \nu}-\frac{\hbar^{2}}{2 m^{*}}\left|\left(\nabla-i \frac{q}{\hbar} \mathbf{A}\right) \psi\right|^{2}-V(r, \psi) .
$$


$\mathcal{L}_{m}$ is a Lagrangian density for matter fields, where $\mu_{0}$ is the permeability of free space, $\hbar$ is Planck's constant, $\mathbf{A}$ is magnetic vector potential, $q$ represents charge, and $m^{*}$ could be the effective mass of a charge in quantum mechanics and simply a constant in relativity. The action $S$ is a functional of a (complex) scalar field $\psi$, and includes the Ricci scalar curvature $\mathcal{R}$, the determinant $g=\operatorname{det}\left(g_{\mu \nu}\right)$, the gravitational coupling constant $\kappa=8 \pi G$, electromagnetic fields $F^{\mu \nu}$ and an interaction potential $V(\psi)$.

\section{Superconducting system}

Our ultimate goal, is to establish a relationship between the $(3+1)$ gravity model in the bulk, and the $(2+1)$ Ginzburg-Landau theory upon the AdS boundary. We incorporate a flexible solution for the potential as follows

$$
V(r, \psi)=\alpha(T) \frac{|\psi|^{2}}{r^{2}}+\chi \frac{|\psi|^{2}}{L^{2}}+\beta \frac{|\psi|^{4}}{2 r^{k}},
$$

where $k$ is an integer (dependent upon the choice of solution), $\chi$ and $\beta$ are constants, and $\alpha(T) \propto\left(T-T_{c}\right)$ is a temperature dependent parameter. It is clear that this parameter $\alpha(T)$ changes sign at some critical temperature $T_{c}$, and corresponds to the phase transition described by the Ginzburg-Landau theory [46-49]. For $T<T_{c}$, this corresponds to a superconducting state; whereas for $T>T_{c}$, this implies a normal state. Therefore, the temperature dependent $\alpha(T)$ provides a significant role for the phase transition. In fact, if $T$ remains constant, then $\alpha(T)=\alpha$ is only a constant coefficient.

In gravity $\chi$ is a classical parameter which describes a square mass of the scalar field, while at the boundary we have a characteristic energy quantised on the scale $L$. This energy may be related to the mass of the scalar particles in the classical theory. The additional term, as $\alpha(T)$, is again related to the mass. It could be interpreted as a new quantity, as the temperature dependent mass deficit. That could be associated with the existence of a black hole since the black hole provides the temperature to the AdS space. The classical quantum transformation may be understood with duality between a classical mass of the scalar particles and the characteristic condensation energy on the quantum boundary.

Figure 2 shows the idea of the phase transition of a superconducting system, associated with symmetry breaking [49]. We also have to mention that our proposed model is not scale invariant due to the choice of potential $V(r, \psi)$.

Generally speaking, superconductors can be classified as either Type I or Type II. The Type I superconductors remain in the Meissner state [50] whilst $H$ is smaller than a critical value $H_{c}$ and as $H$ exceeds $H_{c}$, a normal state emerges. For Type II superconductors, there are the two critical limits, $H_{c 1}$ and $H_{c 2}$. For a magnetic field less than $H_{c 1}$ or greater than $H_{c 2}$, the superconductor is either in the Meissner state or normal state respectively. For vortices nucleating in between the two critical limits, $H_{c 1}<H<H_{c 2}$, we call this a mixed state [3]. For a discussion of vortices in Type II superconductors and holography, see references [51-54].

The BCS theory [47] describes microscopically well all superconductors, and so far is the most used theory. However, in each BCS case we are limited by a specific pairing 


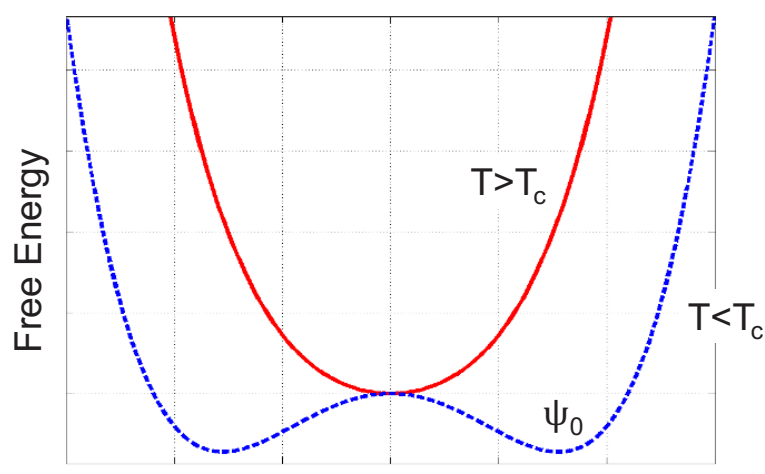

Order Parameter: $\psi$

Superconducting density $|\psi|^{2}$

Figure 2. The free energy associated with a phase transition of superconducting density $|\psi|^{2}$ is shown. For temperature $T>T_{c}$, the minimum free energy is located at $\psi=0-$ corresponding to the normal state $\left(|\psi|^{2}=0\right)$. For $T<T_{c}$, a minimum free energy is now positioned at $\psi=\psi_{0}-$ representing the superconducting state $\left(|\psi|^{2}>0\right)$ which refers to symmetry breaking.

symmetry and the inclusion of a magnetic field leads to nonlinear equations that require complex numerical calculations to be solved. Likewise, the Ginzburg-Landau theory can explain Type II superconducting behaviour near the point of a phase transition in more simple terms of the order parameter. It is due to this fact that Ginzburg-Landau theory is capable of solving the strong coupling of two non-linear differential equations [46]. These coupled equations can resolve the complex scalar field and magnetic potential $(\psi, \mathbf{A})$, respectively. In this study, we focus our attention upon the Type II superconductors for a two-dimensional geometry $[3,55,56]$ (See the appendix for the thermodynamic approach to the derivation of the Ginzburg-Landau equations).

\section{Methodology and analysis}

We will now go on to establish the link of a scalar field $\psi$ of the gravity model in the bulk, to a quantum wave function $\left(\psi=|\psi| e^{i \phi}\right)$ of the Ginzburg-Landau model [3] at the boundary of AdS. We also assume the absence of back-reaction on the infinite boundary $(r \rightarrow \infty)$ of AdS [33] (back-reacting holographic superconductors were discussed in References [57-60]).

We consider a small 2-D superconducting system with an applied static magnetic field $H_{\perp}$ - acting perpendicular to the x-y coordinates at the AdS boundary. We also assume the superconducting state to be stationary, with no overall time dependence of the system. Moreover, we are able to choose the gauge to be $\partial_{x} A_{x}=0$ and $\partial_{y} A_{y}=0$. As such, we can set the parameters $A_{t}=0, A_{r}=0$, with all time and holographic components vanishing also. Therefore, $H_{\perp}=\nabla_{(x, y)} \times \mathbf{A}=\partial_{y} A_{x}-\partial_{x} A_{y}$. Here, $\nabla_{(x, y)}$ is defined as acting upon the x-y coordinate only, with gauge field $\mathbf{A}=\left\{A_{x}, A_{y}\right\}$. The coupled parameters are all functions of coordinates $r, x$ and $y$; these are the magnetic potential $A_{x}(r, x, y), A_{y}(r, x, y)$ and scalar field $\psi(r, x, y)$. 


\subsection{Coupling differential equations}

Following from the Euler-Lagrange equations [61],

$$
\begin{aligned}
& \frac{\partial(\mathcal{L} \sqrt{|g|})}{\partial \psi^{*}}-\frac{d}{d x^{\mu}} \frac{\partial(\mathcal{L} \sqrt{|g|})}{\partial\left(\partial_{\mu} \psi^{*}\right)}=0 \\
& \frac{\partial(\mathcal{L} \sqrt{|g|})}{\partial A_{\mu}}-\frac{d}{d x^{\nu}} \frac{\partial(\mathcal{L} \sqrt{|g|})}{\partial\left(\partial_{\nu} A_{\mu}\right)}=0
\end{aligned}
$$

we obtain the two coupled equations

$$
\begin{aligned}
& \frac{\hbar^{2}}{2 m^{*}}\left(r^{2} s \partial_{r r} \psi+\left(2 r s+r^{2} s^{\prime}\right) \partial_{r} \psi\right)+\frac{\hbar^{2}}{2 m^{*}}\left(\nabla_{(x, y)}-i \frac{q}{\hbar} \mathbf{A}\right)^{2} \psi \\
&-\left(\frac{\alpha(T)}{r^{2}}+\frac{\chi}{L^{2}}+\beta \frac{|\psi|^{2}}{r^{k}}\right) r^{2} \psi=0
\end{aligned}
$$

and

$$
\begin{aligned}
\mathbf{J} & =\frac{1}{r^{2} \mu_{0}} \nabla_{(x, y)} \times \nabla_{(x, y)} \times \mathbf{A}-\frac{1}{\mu_{0}}\left(s^{\prime} \partial_{r} \mathbf{A}+s \partial_{r r} \mathbf{A}\right) \\
& =\frac{q \hbar}{2 m^{*} i}\left(\psi^{*} \nabla_{(x, y)} \psi-\psi \nabla_{(x, y)} \psi^{*}\right)-\frac{q^{2}}{m^{*}} \mathbf{A}|\psi|^{2} .
\end{aligned}
$$

Equation (4.1) is a $2^{\text {nd }}$ order differential equation for the scalar field, whereas eq. (4.2) is for a current density. Both equations incorporate a coupling of two parameters - the vector potential $\mathbf{A}$ and complex scalar field $\psi$. These equations describe the mechanics inside the bulk, where both gravitation and quantum mechanics co-exist [62].

\subsection{Approximated solutions}

Now we consider the complex scalar field $\psi(r, x, y)$, approximated by the following power series $[33-36]$

$$
\psi(r, x, y)=\sum_{n=1}^{\infty} \frac{\psi_{n}(x, y)}{r^{n}} \approx \frac{\psi_{1}(x, y)}{r}+\frac{\psi_{2}(x, y)}{r^{2}}+\ldots,
$$

provided the holographic scale ' $r$ ' is sufficiently large. Since the first two leading terms are linearly independent, one can choose any one of them to be an arbitrary solution of the gravitational system. We can assume $\psi_{1}(x, y) \neq 0$ and $\psi_{2}(x, y)=0$, or otherwise $\psi_{1}(x, y)=0$ and $\psi_{2}(x, y) \neq 0$.

\subsection{At the infinite boundary of AdS space: $r \rightarrow \infty$}

\subsubsection{First scalar solution $\psi_{1} / r\left(\right.$ set $\left.\psi_{2}=0\right)$}

Our approach focuses upon an extreme case where $r \rightarrow \infty$. This is where only quantum mechanics survives at the boundary of AdS space [26, 37]. For a choice of $\psi=\psi_{1} / r$, it follows that $\partial_{r} \psi=-\psi_{1} / r^{2}$ and $\partial_{r r} \psi=2 \psi_{1} / r^{3}$.

Similarly, the magnetic potential $\mathbf{A}$ can be approximated as

$$
\mathbf{A}(r, x, y) \approx\left(1-b \exp \left[-r / r_{0}\right]\right) \mathbf{A}_{1}(x, y),
$$


which is the first order correction for the gauge field, where $b$ is a constant, and hence $\partial_{r} \mathbf{A}=b \mathbf{A}_{1} /\left(r_{0} \exp \left[r / r_{0}\right]\right), \partial_{r r} \mathbf{A}=-b \mathbf{A}_{1} /\left(r_{0}^{2} \exp \left[r / r_{0}\right]\right)$.

For the case of ' $r$ ' tending to a sufficiently large value $\Delta$ (where $\Delta \gg r_{0}$ ), we obtain $s(\Delta)=\Delta^{2} / L^{2}$ and $s^{\prime}(\Delta)=2 \Delta / L^{2}$. The scalar-field equation (4.1) then takes the following form

$$
\frac{\hbar^{2}}{2 m^{*}}\left(\nabla_{(x, y)}-i \frac{q}{\hbar} \mathbf{A}_{1}\right)^{2} \psi_{1}=\left(\frac{\alpha(T)}{\Delta^{2}}+\frac{\chi}{L^{2}}+\frac{1}{L^{2}} \frac{\hbar^{2}}{m^{*}}+\beta \frac{\left|\psi_{1}\right|^{2}}{\Delta^{k+2}}\right) \Delta^{2} \psi_{1}
$$

For the choice of $\chi=-\hbar^{2} / m^{*}$ and the exponent $k=0$, the differential equation (4.1) can reduce to

$$
-\frac{\hbar^{2}}{2 m^{*}}\left(\nabla_{(x, y)}-i \frac{q}{\hbar} \mathbf{A}_{1}\right)^{2} \psi_{1}+\alpha(T) \psi_{1}+\beta\left|\psi_{1}\right|^{2} \psi_{1}=0
$$

which is exactly the same as the $1^{\text {st }}$ non-linear differential equation of Ginzburg-Landau theory [41], where the coherence length is $\xi(T)=\sqrt{\hbar^{2} /\left(2 m^{*}|\alpha(T)|\right)}$ and $\alpha(T)$, in this case, can be approximated as $\alpha(T)=\alpha_{0}\left(T-T_{c}\right) / T_{c}$ near the phase transition.

One of our assumptions is that the influence from the gravity on the AdS (infinity) boundary can be negligible. However, eq. (4.6) still contains some terms relating to the holographic dimension $\left(\Delta^{2}\right)$ on this boundary. Fortunately, this arbitrary choice $\left(\chi=-\hbar^{2} / m^{*}, k=0\right)$ will automatically eliminate the term of $\Delta^{2} / L^{2}$, in which the superconducting system (as governed by quantum mechanics) survives in the absence of gravity. Our choice is similar to some proposals of negative potential $V=-2|\psi|^{2} / L^{2}$ which cancels the $\Delta^{2} / L^{2}$ term [33-36]. We also preserve the phase transition property of the GinzburgLandau model, by introducing $\alpha(T)$ and $\beta$ within the potential $V(\psi)$.

Since the case of $r=\Delta$ is very large and close to the infinite boundary, applying the L'Hospital Rule, eq. (4.2) can reduce to

$$
\begin{aligned}
\mathbf{J} & =\frac{1}{\mu_{0}} \nabla_{(x, y)} \times \nabla_{(x, y)} \times \mathbf{A}_{1} \\
& =\frac{q \hbar}{2 m^{*} i}\left(\psi_{1}^{*} \nabla_{(x, y)} \psi_{1}-\psi_{1} \nabla_{(x, y)} \psi_{1}^{*}\right)-\frac{q^{2}}{m^{*}} \mathbf{A}_{1}\left|\psi_{1}\right|^{2} .
\end{aligned}
$$

Again, this is exactly the same as the $2^{\text {nd }}$ differential equation of Ginzburg-Landau theory, describing the superconducting current [41]. We have now verified that the gravity model on the infinite boundary $(r \rightarrow \infty)$ of AdS, can precisely emulate the Ginzburg-Landau theory in Euclidean space. This means that the superconducting system can be explained on this boundary.

\subsubsection{Second scalar solution $\psi_{2} / r^{2}$ (set $\left.\psi_{1}=0\right)$}

For a choice of $\psi=\psi_{2} / r^{2}$, it follows that $\partial_{r} \psi=-2 \psi_{2} / r^{3}$ and $\partial_{r r} \psi=6 \psi_{2} / r^{4}$. In this case, the possible solution, which satisfies (4.1) and (4.2), is $\mathbf{A}(r, x, y) \approx a \exp \left[-r / r_{0}\right] \mathbf{A}_{2}(x, y)$, where $a$ is a constant, and $\mathbf{A}=0$ as $r \rightarrow \infty$. Therefore, $\partial_{r} \mathbf{A}=a \mathbf{A}_{1} /\left(r_{0} \exp \left[r / r_{0}\right]\right)$, $\partial_{r r} \mathbf{A}=-a \mathbf{A}_{1} /\left(r_{0}^{2} \exp \left[r / r_{0}\right]\right)$.

Similarly, for the choice of $\chi=0$ and the exponent $k=-2$, the differential equation (4.1) can reduce to

$$
-\frac{\hbar^{2}}{2 m^{*}} \nabla_{(x, y)}^{2} \psi_{2}+\alpha(T) \psi_{2}+\beta\left|\psi_{2}\right|^{2} \psi_{2}=0
$$


which is the $1^{\text {st }}$ differential equation of Ginzburg-Landau theory [41], in the absence of a magnetic field $\left(\mathbf{B}=\nabla_{(x, y)} \times \mathbf{A}=0\right)$. As a matter of fact, the solution of $\psi_{2} / r^{2}$ (in the form of a hyperbolic tangent function) can be used to describe the interface between the superconductor and normal metal (superconducting surface). The value $\psi_{2}$ depends on spatial variation on the $\mathrm{x}-\mathrm{y}$ plane, as well as the temperature. This set of solutions indeed explains a special case of temperature dependent GL equations of a superconductor, with a scale of coherence length $\xi(T)$.

\subsection{The physical meaning of $\psi$}

Finally, it is important to discuss about the physical meaning of $\psi$ both in the gravity and superconducting models. As is mentioned above, the scalar field $\psi$ in the bulk (described by the gravity model) now transits to the wave function $\psi=|\psi| \exp (i \theta)$ in the GinzburgLandau model at the AdS boundary. The bulk scalar field $\psi$ is analogue to the order parameter (which also describes the condensation) in the superconducting system. From the literature [33-36, 62, 63], an arbitrary choice of $\psi$ in quantum theory could be either $\psi_{1} / r$ or $\psi_{2} / r^{2}$. In this study, the approximated solution is further focused on $\psi_{1} / r$ with the proposed potential $V(r, \psi)$, in a certain case $\left(\chi=-\hbar^{2} / m^{*}\right.$ and $\left.k=0\right)$. This special choice of solution directly leads to the special coincidence of the gravity model in the AdS, to the superconducting theory at the AdS boundary. This result provides us with confidence to apply the mathematical techniques in quantum gravity to the superconductors (condensed matter physics). We also investigated the solution $\psi_{2} / r^{2}$ - which describes the superconducting density $\left|\psi_{2}\right|^{2}$ near the surface. It provides a special case of the temperature dependence of GL equations without an applied magnetic field.

\section{Conclusion and remarks}

In brief, we have mathematically established the relationship between the $(\mathrm{N}+1)$ dimensional gravity model in the bulk AdS, to the N-dimensional Ginzburg-Landau system at the infinity Ads boundary $(r \rightarrow \infty)$. It is found that the two coupled differential GinzburgLandau equations at the Ads boundary can be derived from the equations of motion residing inside the bulk of AdS space. We restrict our efforts to the two-dimensional, asymptotically flat spatial domain of AdS space (as $r \rightarrow \infty)$, and also propose the potential $V(r, \psi)$ for a special solution. The quantum gravity model is thought to expose many features that appear in the quantum critical electrons in the cuprate superconductors. As such, simplified models to analyse the nature of the condensed state using the AdS to GinzburgLandau formulation could lead to valuable new insights both in superconductivity, and black-hole physics. It is also worth noting that Landau theory provides a unifying language for describing continuous phase transitions and critical phenomena in a plethora of physical systems $[23,64,65]$. For example, ferro- and antiferromagnets, fluid mixtures, oscillators and superfluids are other systems that exhibit transitions from symmetric high temperature phases to low temperature ordered ones at a critical temperature. Thus, for analogous Landau systems an expansion of the free energy in terms of the order parameter 
and adoption of the current methodology may provide valuable information to understand complex behaviour.

\section{A Ginzburg-Landau equations from thermodynamic approach}

The thermodynamic derivations of the two Ginzburg-Landau (GL) equations are shown in this section $[46,47,49]$. We restrict our case to 2-D (on the $\mathrm{x}$-y plane), and assume the magnetic field $\mathbf{B}=\nabla_{(x, y)} \times \mathbf{A}$ lies along with $\mathrm{z}=$ axis, where $\mathbf{A}$ is a magnetic potential. The total free energy of a system in the superconducting state $F_{s}$ is

$$
F_{s}=F_{n}+\int\left(\frac{\hbar^{2}}{2 m^{*}}\left|\left(\nabla_{(x, y)}-i \frac{q}{\hbar} \mathbf{A}\right) \Psi\right|^{2}+\Delta(T)|\Psi|^{2}+\frac{\eta}{2}|\Psi|^{4}\right) \mathrm{d}^{2} \mathrm{x},
$$

where $F_{n}$ is the free energy in a normal state, $m^{*}$ is the effective mass of a Cooper pair, $\Psi$ is a superconducting wave function, $\Delta(T) \sim(T c-T) / T_{c}$ is a function depending on temperature, $T_{c}$ is a critical temperature of the system, and $\eta$ is a parameter dependent upon the material. By small variation of $F_{s}\left(\delta F_{s}\right)$ with respect to $\delta \Psi$ and $\delta \mathbf{A}$ correspondingly, one can obtain the $1^{\text {st }}$ GL equation (see $[46,47,49]$ )

$$
-\frac{\hbar^{2}}{2 m^{*}}\left(\nabla_{(x, y)}-i \frac{q}{\hbar} \mathbf{A}\right)^{2} \Psi+\Delta(T) \Psi+\eta|\Psi|^{2} \Psi=0,
$$

and the $2^{\text {nd }}$ GL equation

$$
\mathbf{J}=\frac{q \hbar}{2 m^{*} i}\left(\Psi^{*} \nabla_{(x, y)} \Psi-\Psi \nabla_{(x, y)} \Psi^{*}\right)-\frac{q^{2}}{m^{*}} \mathbf{A}|\Psi|^{2} .
$$

Open Access. This article is distributed under the terms of the Creative Commons Attribution License (CC-BY 4.0), which permits any use, distribution and reproduction in any medium, provided the original author(s) and source are credited.

\section{References}

[1] J.G. Bednorz and K.A. Muller, Possible high Tc superconductivity in the Ba-La-Cu-O system, Z. Phys. B 64 (1986) 189 [INSPIRE].

[2] L.P. Gor'kov, Microscopic derivation of the Ginzburg-Landau equations in the theory of superconductivity, Sov. Phys. JETP 36 (1959) 1364.

[3] A.A. Abrikosov, Nobel Lecture: Type-II superconductors and the vortex lattice, Rev. Mod. Phys. 76 (2004) 975 [InSPIRE].

[4] M. Fratini et al., Scale-free structural organization of oxygen interstitials in $\mathrm{La}_{2} \mathrm{CuO}_{4+y}$, Nature 466 (2010) 841 [arXiv: 1008.2015].

[5] F.V. Kusmartsev, D.D. Castro, G. Bianconi and A. Bianconi, Transformation of strings into an inhomogeneous phase of stripes and itinerant carriers, Phys. Lett. A 275 (2000) 118.

[6] F.V. Kusmartsev and M. Saarela, Dipolar clusters and ferroelectricity in high $T_{c}$ superconductors, Int. J. Mod. Phys. B 29 (2015) 1542002. 
[7] D. van de Marel et al., Quantum critical behaviour in a high-tc superconductor, Nature 425 (2003) 271 [INSPIRE].

[8] G.T. Horowitz, From Gravity to Thermal Gauge Theories: The AdS/CFT Correspondence, Chapter 10: Introduction to Holographic Superconductors, E. Papantonopoulos eds., Spinger-Verlag, Heidelberg Germany (2011).

[9] J. Zaanen, High-temperature superconductivity: The benefit of fractal dirt, Nature 466 (2010) 825.

[10] P.W. Anderson, "confinement" in the one-dimensional Hubbard model: Irrelevance of single-particle hopping, Phys. Rev. Lett. 67 (1991) 3844.

[11] A. Nersesyan, A. Luther and F. Kusmartsev, Scaling properties of the two-chain model, Phys. Lett. A 176 (1993) 363.

[12] S.A. Kivelson, E. Fradkin and V.J. Emery, Electronic liquid-crystal phases of a doped Mott insulator, Nature 393 (1998) 550

[13] F. Kusmartsev, A. Luther and A. Nersesyan, Theory of a 2D Luttinger liquid, JETP lett. 55 (1992) 724.

[14] F.V. Kusmartsev, Formation of electron strings in narrow band polar semiconductors, Phys. Rev. Lett. 84 (2000) 530.

[15] S. Lederer, Y. Schattner, E. Berg and S. Kivelson, Enhancement of superconductivity near a nematic quantum critical point, Phys. Rev. Lett. 114 (2015) 097001 [arXiv:1406.1193].

[16] S.W. Hawking, M.J. Perry and A. Strominger, Soft hair on black holes, Phys. Rev. Lett. 116 (2016) 231301 [arXiv:1601.00921] [INSPIRE].

[17] S.W. Hawking and D.N. Page, Thermodynamics of Black Holes in anti-de Sitter Space, Commun. Math. Phys. 87 (1983) 577 [inSPIRE].

[18] J.L.F. Barbon and E. Rabinovici, Extensivity versus holography in anti-de Sitter spaces, Nucl. Phys. B 545 (1999) 371 [hep-th/9805143] [INSPIRE].

[19] J.B. Hartle and S.W. Hawking, Solutions of the Einstein-Maxwell equations with many black holes, Commun. Math. Phys. 26 (1972) 87 [INSPIRE].

[20] J. Erdmenger, X.-H. Ge and D.-W. Pang, Striped phases in the holographic insulator/superconductor transition, JHEP 11 (2013) 027 [arXiv:1307.4609] [INSPIRE].

[21] G. Dvali and C. Gomez, Black Holes as Critical Point of Quantum Phase Transition, Eur. Phys. J. C 74 (2014) 2752 [arXiv:1207.4059] [INSPIRE].

[22] K. Hashimoto et al., A sharp peak of the zero-temperature penetration depth at optimal composition in $\mathrm{BaFe}_{2}\left(A s_{1-x} P_{x}\right)_{2}$, Science 336 (2012) 1554.

[23] K. Jin et al., Anomalous magnetoresistance in the spinel superconductor $\mathrm{LiTi}_{2} \mathrm{O}_{4}$, Nature Commun. 6 (2015) 7183

[24] T. Faulkner, N. Iqbal, H. Liu, J. McGreevy and D. Vegh, Strange metal transport realized by gauge/gravity duality, Science 329 (2010) 1043 [INSPIRE].

[25] W.H. Zurek, Cosmological Experiments in Superfluid Helium?, Nature 317 (1985) 505 [INSPIRE].

[26] J. Maldacena, The illusion of gravity, Sci. Am. 293 (2005) 57.

[27] Z. Merali, String theory finds a bench mate, Nature 478 (2011) 302 [INSPIRE]. 
[28] R. Bousso and J. Polchinski, The string theory landscape, Sci. Am. 291 (2004) 78.

[29] F. Wilczek, QCD made simple, Phys. Today 53 (2000) 22.

[30] L. Yin, D. Hou and H.-c. Ren, Ginzburg-Landau theory of a holographic superconductor, Phys. Rev. D 91 (2015) 026003 [arXiv:1311.3847] [INSPIRE].

[31] A. Adams, P.M. Chesler and H. Liu, Holographic Vortex Liquids and Superfluid Turbulence, Science 341 (2013) 368 [arXiv: 1212.0281] [InSPIRE].

[32] J.B. Hartle, S.W. Hawking and T. Hertog, Quantum Probabilities for Inflation from Holography, JCAP 01 (2014) 015 [arXiv: 1207.6653] [INSPIRE].

[33] S.S. Gubser, Breaking an Abelian gauge symmetry near a black hole horizon, Phys. Rev. D 78 (2008) 065034 [arXiv:0801.2977] [InSPIRE].

[34] G.T. Horowitz and M. M. Roberts, Holographic superconductors with various condensates, Phys. Rev. D 78 (2008) 126008 [arXiv:0810.1077] [INSPIRE].

[35] S.A. Hartnoll, C.P. Herzog and G.T. Horowitz, Building a Holographic Superconductor, Phys. Rev. Lett. 101 (2008) 031601 [arXiv:0803.3295] [INSPIRE].

[36] A.M. Garcia-Garcia, J.E. Santos and B. Way, Holographic Description of Finite Size Effects in Strongly Coupled Superconductors, Phys. Rev. B 86 (2012) 064526 [arXiv: 1204.4189] [INSPIRE].

[37] I.R. Klebanov and J.M. Maldacena, Solving quantum field theories via curved spacetimes, Phys. Today 62 (2009) 28.

[38] A. Bagchi, R. Basu, D. Grumiller and M. Riegler, Entanglement entropy in Galilean conformal field theories and flat holography, Phys. Rev. Lett. 114 (2015) 111602 [arXiv: 1410.4089] [INSPIRE].

[39] R. Bousso, The holographic principle for general backgrounds, Class. Quantum Grav. 17 (2000) 852.

[40] R. Bousso, The holographic principle, Rev. Mod. Phys. 74 (2002) 825 [hep-th/0203101].

[41] V.L. Ginzburg, Nobel Lecture: On superconductivity and superfluidity (what I have and have not managed to do) as well as on the 'physical minimum' at the beginning of the XXI century, Rev. Mod. Phys. 76 (2004) 981 [INSPIRE].

[42] N.R.F. Braga, Quantum fields in anti-de Sitter space and the Maldacena conjecture, Braz. J. Phys. 32 (2002) 880.

[43] M. Montull, A. Pomarol and P.J. Silva, The Holographic Superconductor Vortex, Phys. Rev. Lett. 103 (2009) 091601 [arXiv:0906.2396] [INSPIRE].

[44] C.P. Herzog, An Analytic Holographic Superconductor, Phys. Rev. D 81 (2010) 126009 [arXiv: 1003.3278] [INSPIRE].

[45] K. Maeda, M. Natsuume and T. Okamura, Vortex lattice for a holographic superconductor, Phys. Rev. D 81 (2010) 026002 [arXiv:0910.4475] [INSPIRE].

[46] M. Tinkham, Introduction to Superconductivity, Dover Publication, New York U.S.A. (1996).

[47] D. Saint James and P.G.D. Gennes, Onset of superconductivity in decreasing fields, Phys. Lett. 7 (1963) 306.

[48] V. Schweigert and F. Peeters, Influence of the confinement geometry on surface superconductivity, Phys. Rev. B 60 (1999) 3084 [cond-mat/9905016]. 
[49] J.F. Annett, Superconductivity, Superfluids and Condensates, Oxford University Press, New York U.S.A. (2004).

[50] A.T. Dorsey, Superconductivity: Geometry spawns vortices, Nature 408 (2000) 783.

[51] D. Roychowdhury, Chern-Simons vortices and holography, JHEP 10 (2014) 018 [arXiv: 1407.3464].

[52] N. Banerjee, S. Dutta and D. Roychowdhury, Chern-Simons Superconductor, Class. Quant. Grav. 31 (2014) 245005 [arXiv:1311.7640] [INSPIRE].

[53] D. Roychowdhury, Towards holographic duals for anomalous supercurrents, arXiv: 1403.0085 [INSPIRE].

[54] M. Rogatko and K.I. Wysokinski, Holographic vortices in the presence of dark matter sector, JHEP 12 (2015) 041 [arXiv:1510.06137] [INSPIRE].

[55] L. Chibotaru, A. Ceulemans, V. Bruydoncx and V. Moshchalkov, Symmetry-induced formation of antivortices in mesoscopic superconductors, Nature 408 (2000) 833.

[56] A. Mel'nikov, I. Nefedov, D. Ryzhov, I. Shereshevskii, V. Vinokur and P. Vysheslavtsev, Vortex states and magnetization curve of square mesoscopic superconductors, Phys. Rev. B 65 (2002) 140503 [InSPIRE].

[57] Ł. Nakonieczny and M. Rogatko, Analytic study on backreacting holographic superconductors with dark matter sector, Phys. Rev. D 90 (2014) 106004 [arXiv:1411.0798] [INSPIRE].

[58] Ł. Nakonieczny, M. Rogatko and K.I. Wysokinski, Magnetic field in holographic superconductor with dark matter sector, Phys. Rev. D 91 (2015) 046007 [arXiv:1502.02550] [INSPIRE].

[59] Ł. Nakonieczny, M. Rogatko and K.I. Wysokiński, Analytic investigation of holographic phase transitions influenced by dark matter sector, Phys. Rev. D 92 (2015) 066008 [arXiv: 1509.01769] [INSPIRE].

[60] M. Rogatko and K.I. Wysokinski, P-wave holographic superconductor/insulator phase transitions affected by dark matter sector, JHEP 03 (2016) 215 [arXiv:1508.02869] [INSPIRE].

[61] C.L. Lewis, Explicit gauge covariant Euler-Lagrange equation, Am. J. Phys. 77 (2009) 839 [arXiv:0907.2301] [INSPIRE].

[62] A.G. Green, An introduction to gauge-gravity duality and its application in condensed matter, Contemp. Phys. 54 (2013) 33 [arXiv:1304.5908].

[63] R. Gregory, S. Kanno and J. Soda, Holographic Superconductors with Higher Curvature Corrections, JHEP 10 (2009) 010 [arXiv: 0907.3203] [INSPIRE].

[64] D.M. Forrester, K.E. Kürten and F. V. Kusmartsev, Fractal metamaterials composed of electrically isolated $\pi$-rings, Sci. Lett. J. 4 (2015) 133.

[65] L. Kramer, F. Hynne, P. Graae So/renson and D. Walgraef, The Ginzburg-Landau approach to oscillatory media, Chaos 4 (1994) 443. 